\title{
ON THE PROBLEM OF OBSERVABILITY IN THE THEORY OF TRANSIENT EMISSION SPECTRA
}

\author{
P. SAARI. MOODETAVUSEST KIIRGUSE OLEMINEKUSPEKTRITE TEOORIAS \\ П. СААРИ, УЧЕТ ТРЕБОВАНИЯ ИЗМЕРЯЕМОСТИ В ТЕОРИИ ПЕРЕХОДНЫХ СПЕКТРОВ \\ СВЕЧЕНИЯ
}

Hand in hand with the growth of the potentialities of experiment in recent years, the problem of the classification of the RSE components has become a subject of new discussions (see refs. in $\left[^{1}\right]$ ). In particular, a number of papers (see refs. in $\left[{ }^{2}\right]$ ) consider the time dependence of the frequency-integrated intensity of RSE. The greatest hopes in the study of relaxation processes and in separation of the RSE components, above all scattering and hot luminescence $\left[{ }^{3,1}\right]$, are pinned on the investigation of the RSE spectra with subpicosecond time resolution. Some calculations of such transient spectra have been performed already for various models of luminescence centres $\left[^{4-6}\right]$. However, here arises the question of the physical sense of simultaneous time and spectral resolution.

It is well known that the calculation of an emission spectrum reduces to an evaluation of the mean value of the photon number operator ( $\Omega$ being the photon frequency):

$$
W(\Omega, t) \propto\left\langle n_{\Omega}\right\rangle, n_{\Omega}=a_{\Omega}^{+} a_{\Omega} .
$$

In the case of a steady-state problem the dependence of (1) on the time $t$ is linear and the observable spectrum is given as a derivative of (1) with respect to $t$. Similarly, one can express the transient spectrum in a nonstationary case: $\Pi(\Omega, t)=d W / d t$. It is just the quantity which has been calculated in papers $\left[{ }^{4-6}\right]$. However, the quantity $\Pi$ turns out not to be directiy observable. It may take negative values and it does not give the experimentaliy observable photon counting rate at the frequency $\Omega *$. In connexion with that, the authors of $\left[^{4-6}\right]$ have mentioned a necessity of carrying out some smoothing procedure of the expressions obtained. But as this procedure is unperformable afterwards we develop here an approach to the calculation of the transient RSE spectra, which from the very beginning takes into account the photodetection measurement process.

According to $\left[{ }^{8}\right]$, the observable photon counting rate at a point $r, t$ of the field is determined by the correiation function $G(r, t, \tau)=$ $=\left\langle E^{-}(r, t) E^{+}(r, t-1-\tau)\right\rangle$ at $\tau=0$, where $E^{-}$and $E^{+}$are the negativeand positive-frequency components of the electric field operator, respec-

* According to $\left[{ }^{7}\right]$, even positively definite integral of $\Pi$ over $\Omega-$ the energy flow rate into the field - contains unobservable effects. 
tively. In our case the Hamiltonian includes not only the energy of the free field but also the energies of the emitting system, of the field exciting the latter, and of corresponding interactions. The initial conditions are formulated for the moment when the leading front of the exciting pulse, which always exists in a real experiment, reaches the emitting system. Let now the field emitted by the system at $r=0$ act via spectrally selective filter** on a photodetector placed at $r$. Then the field falling onto the detector may be expressed as ***

$$
E_{f}(r, t)=\int F\left(t-t^{\prime}\right) E\left(r, t^{\prime}\right) d t^{\prime},
$$

where $F$ is the filter pulse response, and the photon counting rate measured behind the filter is given by

$$
{ }^{3} I_{f}(r, t)=\iint F^{*}\left(t-t^{\prime}\right) F^{\prime}\left(t-t^{\prime}-\tau\right) G\left(r, t^{\prime}, \tau\right) d t^{\prime} d \tau \text {. }
$$

For the case of a steady-state problem, when $G$ does not depend on $\tau$, the Fourier transformation of (3) results in an expression of the spectrum convolution with the instrument response function, which is familiar in spectroscopy. In the particular case of a spectral instrument with normal slit width one can put $F(t)=T^{-1}[Y(t)-Y(t-$ $-T)] \exp (-i \Omega t)$, where $Y$ is the Heavyside unit step and $\Omega$ is the spectrum frequency under examination. $T$ determines the integration interval of the field in (3), i. e., the spectral resolution (e. g., it equals to the interval between the waves coming from the right and left edges of the grating, or to the exposure time in case the time resolution is accomplished by a light shutter). Evaluating (3) to the second order in the perturbation theory, we have (neglecting the smooth frequency dependences) for the RSE time-dependent photon counting rate detected at the frequency $\Omega$ behind the spectral instrument:

$$
I_{T}^{Q}(t)=\frac{1}{T^{2}} \int_{0}^{t} \int_{0}^{t} d t_{1} d t^{\prime}{ }_{1} Y\left(t_{1}-t+T\right) Y\left(t^{\prime}{ }_{1}-t+T\right) \mathrm{e}^{i \Omega\left(t_{1},-t_{1}\right)} \int_{0}^{t_{1}} \int_{0}^{t_{4}^{\prime}} d t_{2} d t^{\prime}{ }_{2} K G_{e},
$$

where

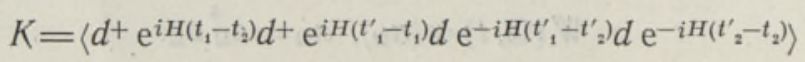

is the correlation function of the emitting material system at thermal equilibrium which has been evaluated in $\left[{ }^{9,4-6}\right]$ for various models of a luminescence centre, $d$ and $H$ are the dipole momentum and energy operators, respectively ( $H$ includes the radiative damping operator - i as a requisite correction from higher orders [5])、 $G_{e} \equiv G_{e}\left(t_{2}, t_{2}^{\prime}-t_{2}\right)$ is the correlation function of the exciting field at $r=0$. The time required for the light to traverse from the emitter to detector is subtracted from $t$.

Eq. (4) incorporates the alternative between the frequency and time measurements: if $T \rightarrow 0$, time resolution ever increases but the spectral resolution of the emission vanishes; on the contrary, if $T \rightarrow \infty Y$-functions fall out and $I_{T}{ }^{2}(t)$ converts to $W(\Omega, t)\left[{ }^{5}\right]$ - the spectrum of all photons in the field emitted during the interval $0 \div t$. We reach similar conclusions when considering spectrally-selective detection by means of a two-level

of interest.

** The detector's sensitivity is assumed to be constant in the frequency region

*** An expansion of the field into plane waves (and raduction of the problem to a one-dimensional one) is implied. If working with normal modes which take into account the existence of the filter, then (2) has already been incorporated in the expansion. 
absorber. On the two-level models working without any mode expansion of the field, it is easy to make sure that the typical oscillatory behaviours of transient spectra are inherent in both near- and far-zone fields.

A calculation of the quantity $I_{T}^{2}(t)$ for the modulation-broadened twolevel model $\left[{ }^{10}\right]$ as a probe model in the RSE problem shows that in contrast to the calculations of the spectrum $\Pi(\Omega, t)\left[{ }^{6}\right]$, the time and frequency criteria of a distinction between scattering and luminescence are alternative at resonance. Let us mention that the spectrum $\Pi$ can be, in principle, correlated with an experiment as a derivative of counting rate behind a super narrow-band spectral filter with respect to time; however, after the spectrum $W$ (or $\Pi$ ) has been calculated, it is impossible to take into account the finite band width of the filter by a convolution of the result with a function depending only on the filter characteristics. The reason is that $W$ and $\Pi$ are the functions of one time argument, i.e., the evaluation of these quantities passes over from amplitudes to probabilities with no regard to the measurement procedure. In conclusion, the computer calculations of transient spectra, of essence in the further progress in the RSE problem, should be carried out, as it follows from (4), in the form which allows taking into account a given instrument before the phase of photons is eliminated. If, in addition, the finite time resolution of the recording of photoelectron emission from (nonselective) detector has to be incorporated, the phase problem does not arise, and it is sufficient to take the convolution of the spectra with a corresponding pulse response. Further generalizations of Eq. (4): in accordance with (3) for an arbitrary filter, the $Y$-function should be replaced by a corresponding response function, e. g., by $Y(X) x^{-1} \sin \Delta x$ in the case of spectral slit width $\Delta$ wider than the normal one. Especially in the latter case, the measurement of a coherent (stimulated) emission possible at picosecond laser excitation needs particular consideration. The inhomogeneity of the spectrum and dimensions of emitting volume should also be taken into consideration.

The author is indebted to $\mathrm{K}$. Rebane and J. Kikas for valuable remarks, and also to V. Hizhnyakov for stimulating discussions.

\section{REFERENCES}

1. Р ебан е К. К., С а ар и П. М., Изв. АН СССР, сер. физ., 40, 1778 (1976); J. Luminescence, 12/13, 23 (1976). ibid. (1977) (to be published).

2. Mukamel, S., Ben-Reuven, A., Jortner, J., J. Chem. Phys., 64, 3971 (1976).

3. S a a ri, P., Rebane, K., Solid State Comm., 7, 887 (1969).

4. To y o z a wa, Y., Phys. Soc. Japan, 41, 400 (1976).

5. Хи жняков В., Реб ауне И., Изв. АН ЭССР, Физ. Матем., 26, 260 (1977); ЖЭТФ (в печати).

6. T a k a g a ha r a, T., Thesis, Tokyo, 1976.

7. Khoo, I. C., E berly, J. H., Phys. Rev. A, 14, 2174 (1976).

8. G l a u be r, R. J.; Phys. Rev., 130, 2529 (1963).

9. Hizhnyakov, V., Tehver, I., Phys. stat. sol., 21, 755 (1967).

10. H u b e r, D. L., Phys. Rev., 178, 93 (1969). 